\title{
Herbig-Haro jets in 3D: the HL/XZ Tauri region $\star, \star \star$
}

\author{
T. A. Movsessian ${ }^{1}$, T. Yu. Magakian ${ }^{1}$, J. Bally ${ }^{2}$, M. D. Smith ${ }^{3}$, A. V. Moiseev ${ }^{4}$, and S. N. Dodonov ${ }^{4}$ \\ ${ }^{1}$ Byurakan Astrophysical Observatory, 378433 Aragatsotn reg., Armenia \\ e-mail: tigmov@bao.sci.am, tigmag@sci.am \\ 2 University of Colorado, USA \\ e-mail: John.Bally@Colorado.EDU \\ ${ }^{3}$ Centre for Astrophysics and Planetary Science, University of Kent, Canterbury, CT2 7NH, UK \\ e-mail: m.d.smith@kent.ac.uk \\ 4 Special Astrophysical Observatory, N.Arkhyz, Karachaevo-Cherkesia, 369167, Russia \\ e-mail: moisav@sao.ru,dodo@sao.ru
}

Received 25 January 2007 / Accepted 13 April 2007

\section{ABSTRACT}

\begin{abstract}
Context. Jets and outflows from young stellar objects (YSOs) can be identified and traced through the distribution and structure of shocked emission-line features. To understand the formation of these regions requires kinematic data at high spectral resolution and with full spatial coverage.

Aims. In this work, we investigate the environment of HL/XZ Tau, which contains a compact and very active nest of YSOs. We explore the kinematic properties of the close association of jets in this region and study the interaction of jets with the ambient medium, as well as the outflows with each other.

Methods. We present scanning Fabry-Perót interferometry of the HL/XZ Tau region in H $\alpha$ and [S II] $6716 \AA$ emission. We also measure the proper motions of the knots in the outflows, as derived from images obtained in 1997 and 2001, to achieve the full 3D kinematic picture.

Results. Radial velocities of the HL Tau jet indicate a fast spine of low excitation surrounded by a slower sheared sheath of higher excitation. Proper motions range from $200-220 \mathrm{~km} \mathrm{~s}^{-1}$ in the HL Tau jet and are aligned within 10 degrees of the jet spine. In combination, the proper motions and radial velocities indicate that three outflows in this region may be interacting with each other. Evidence of an outflow associated with $\mathrm{LkH} \alpha 358$ is found, and we suggest it is a source of Herbig-Haro (HH) knots that lie to the southeast of HL Tau and HH 265.

Conclusions. We conclude that the southern lobe of the XZ Tau wind disrupts the eastern lobe of the collimated outflow from $\mathrm{LkH} \alpha 358$. The jet emerging from HL Tau is deflected by the northern lobe of the wind from XZ Tau. We propose several probable explanations for the unusual structure of the HL Tau jet. It is plausible that the shocks in the jet spine are maintained by the ram pressure of a low-density crosswind from XZ Tau. The crosswind interacts to form a sheath of entrained gas.
\end{abstract}

Key words. stars: formation - ISM: jets and outflows - ISM: clouds

\section{Introduction}

A small group of young stellar objects is located in the northwest region of the well-studied dark cloud L1551, which lies in the nearby $(140 \mathrm{pc})$ Taurus-Auriga star formation region. In close association with this group are several jet and outflow sources within a field of size $0.05 \times 0.1 \mathrm{pc}$ (Mundt et al. 1988). This extraordinary nest includes two bright pre-main sequence stars, HL Tau and XZ Tau, and HH 30 - a young star with an edge-on oriented circumstellar disk. Another young object is the emission-line star $\mathrm{LkH} \alpha 358$.

Well-collimated optical flows in this region were among the first stellar jets to be found (Mundt \& Fried 1983). Thorough optical imaging (Mundt et al. 1988) and spectroscopy

\footnotetext{
* Based partly on observations collected with the $6 \mathrm{~m}$ telescope of the Special Astrophysical Observatory (SAO) of the Russian Academy of Sciences (RAS), operated under the financial support of the Science Department of Russia (registration number 01-43.).

$\star \star$ Data cubes with the results of FP interferometry are available in electronic form at the CDS via anonymous ftp to cdsarc.u-strasbg.fr (130.79.128.5) or via http://cdsweb.u-strasbg.fr/cgi-bin/qcat?J/A+A/470/605
}

(Mundt et al. 1990) of this field revealed bipolar jets emanating from HL Tau, XZ Tau, and HH 30.

In addition, an elongated object with an emission-line spectrum corresponding to shocked gas, known as the $\mathrm{H} \alpha$ jet, has no known source. The unusual morphology and spectral features of the HL Tau jet led to the suggestion that two intersecting jets are being observed. An optically invisible radio source, VLA 1, was proposed to be the driving source for the second jet (Brown et al. 1985). No evidence was found of an optically detectable outflow from $\mathrm{LkH} \alpha 358$.

The radial velocities of the optical flows clearly show the bipolar nature of the HL Tau and XZ Tau jets. The radial velocities in the HH 30 jet are nearly equal on both sides of the star, indicating an alignment with the plane of the sky. Furthermore, the complex nature of the velocity field in the VLA 1 jet with high and low velocity components was revealed (Mundt et al. 1990).

Further observations in the near and far infrared (IRAS and 2MASS surveys), as well as in the radio (Rodriguez et al. 1992, 1994; Wilner et al. 1996), did not confirm the VLA 1 source. Thus, its existence now seems doubtful (see, e.g., Rodriguez et al. 1994), and one must assume that the unusual features of 
the HL Tau jet, which we describe in detail, result from its specific characteristics and not from the combination of two jets.

Several $\mathrm{HH}$ objects also lie up to $4-5^{\prime}$ northeast of HL Tau (Lopez et al. 1995). They exhibit large proper motions (Lopez et al. 1996). The location of these features indicates that they are driven to their present positions by either HH 30 or the HL Tau jet. However, the material could be swept-up and compressed gas from the XZ Tau wind. The proper motion vectors do not distinguish between these alternatives.

In this paper, we present data from new Fabry-Pérot (FP) scanning interferometry observations in the $\mathrm{H} \alpha$ and [S II] emission lines. The HL/XZ Tau region was fully covered. Similar to other 3D methods, FP scanning interferometer provides detailed spectrophotometric information alongside full spatial coverage, but the spectral range is usually limited to one spectral line. However, its distinct advantage is a wide field of view, combined with high spectral resolution, ideal for studying extended emission objects such as Herbig-Haro (HH) jets.

To obtain the full three-dimensional picture of the jet kinematics, we measured proper motions of all knots where such measurements were possible.

\section{Observations and data reduction}

\subsection{FP scanning interferometry}

Observations in the $\mathrm{H} \alpha$ emission line were carried out in good conditions at the prime focus of the $6 \mathrm{~m}$ SAO telescope on 17 September 2001 (seeing was $1-1.5^{\prime \prime}$ ). We used a scanning Fabry-Pérot interferometer placed in the collimated beam of the SCORPIO focal reducer (Afanasiev \& Moiseev 2005). The SCORPIO capabilities for FP interferometry were described by Moiseev (2002). The detector was a TK1024 $1024 \times 1024$ pixel CCD array. The observations were performed with $2 \times 2$ pixel binning to reduce the read-out time, so $512 \times 512$ pixel images were obtained in each spectral channel. The field of view was $4.8^{\prime}$ for a scale of $0.56^{\prime \prime}$ per pixel. An interference filter with $F W H M=15 \AA$ centered on the $\mathrm{H} \alpha$ line cut the desired spectral range.

For the observations, we used a Queensgate ET-50 interferometer operating in the 501st order of interference (at the $\mathrm{H} \alpha$ wavelength), providing a spectral resolution of $F W H M \approx$ $0.8 \AA\left(\right.$ or $\sim 36 \mathrm{~km} \mathrm{~s}^{-1}$ ) for a range of $\Delta \lambda=13 \AA$ (or $\sim 590 \mathrm{~km} \mathrm{~s}^{-1}$ ) free from order overlapping. The number of spectral channels was 36 and the size of a single channel was $\Delta \lambda \approx 0.36 \AA$ $\left(\sim 16 \mathrm{~km} \mathrm{~s}^{-1}\right)$.

The observations in the $\lambda 6716 \AA$ [S II] line were carried out with the same SCORPIO equipment on 30 January 2004 and in a similar way. However, in this case, we used the $2 \mathrm{~K} \times 2 \mathrm{~K}$ detector EEV 42-40 operated with $4 \times 4$ pixel binning. The field of view was $6^{\prime}$ sampled with a scale $0.70^{\prime \prime}$ per pixel. This time the seeing was $1.5-2^{\prime \prime}$.

We reduced the interferometric data with IDL-based software developed at the SAO (Moiseev 2002). After the primary data reduction, the subtraction of night-sky lines, and wavelength calibration, the observational material represents "data cubes" in which each point in the $512 \times 512$ pixel field contains a 36-channel spectrum.

\subsection{Proper motions}

Proper motions were determined using images obtained in 1997 and 2001 with the Mosaic CCD camera on the Mayall $4 \mathrm{~m}$ telescope at the Kitt Peak National Observatory. Motions were

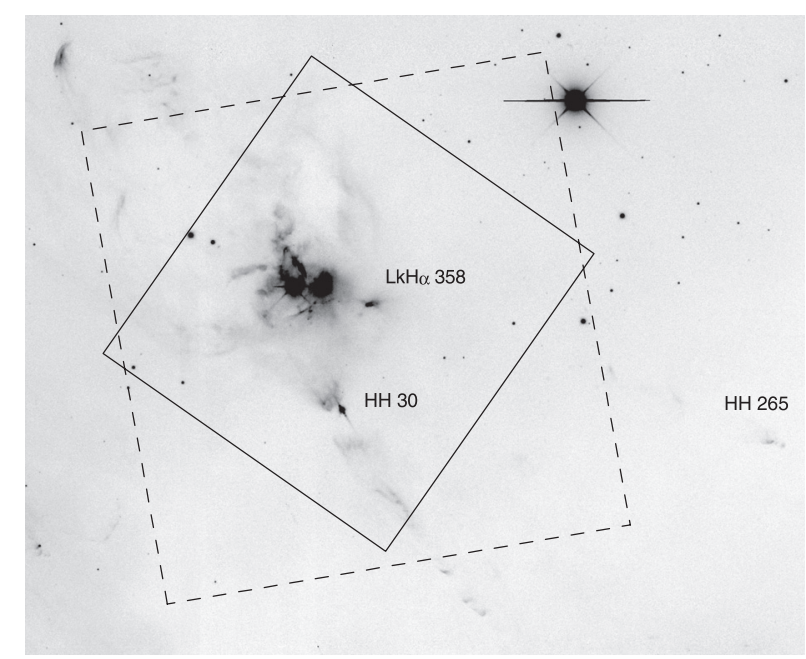

Fig. 1. The $\mathrm{H} \alpha$ image of the northeastern part of the L1551 molecular cloud, obtained with the Mosaic system on the $4 \mathrm{~m}$ KPNO telescope. The regions covered by FP interferometry are outlined with rectangles solid for $\mathrm{H} \alpha$ and dashed for [S II]. HL Tau (right) and XZ Tau (left) are the pair of bright stars near the center of the rectangles.

determined using the "difference squared" method described in Hartigan et al. (2001) and Reipurth et al. (2002). This algorithm compares the extended emission in a selected rectangular region in the first-epoch image with the corresponding emission in the second-epoch image, but shifted in the plane-of-the-sky by a vector that minimizes the differences between the selected region. As in the case of FP interferometry, proper motions were obtained separately for $\mathrm{H} \alpha$ and [S II] emission lines.

\section{Results of FP interferometry}

The field of view of the SCORPIO camera covered the region shown in Fig. 1. All the outflow sources and $\mathrm{HH}$ jets around HL/XZ Tau fall within the data cube, allowing us to get the entire kinematic picture of this region. Our results are in good agreement with the previous slit spectroscopy (Mundt et al. 1987, 1988, 1990; Magakian et al. 1989), but we uncovered many new interesting details, especially concerning the HL Tau flow. Below, we present the new results and discuss the objects individually.

\subsection{The HL Tau jet}

\subsection{1. $\mathrm{H} \alpha$ emission}

The HL Tau jet is narrow and more prominent in the highvelocity channels with several well-defined knots that trace a broken line as shown in the channel maps presented in Fig. 2. The first 15 arcsec of the jet between HL Tau and the first bend is faint; however, the knots located immediately downstream in the middle part of the flow are bright. The counterjet, described by Mundt et al. (1990), is too faint in $\mathrm{H} \alpha$ to be detected. The middle part of the HL Tau jet, previously ascribed to the VLA 1 flow, undergoes variations in morphology that correlate with changes in radial velocity. The jet is relatively narrow in the high-velocity channels, while at low velocities it is considerably wider with regular arcuate structures, which trace the locations of highvelocity knots. Overall, the jet at low velocities can be described as a cone with helical arms wound tightly on the cone surface. 

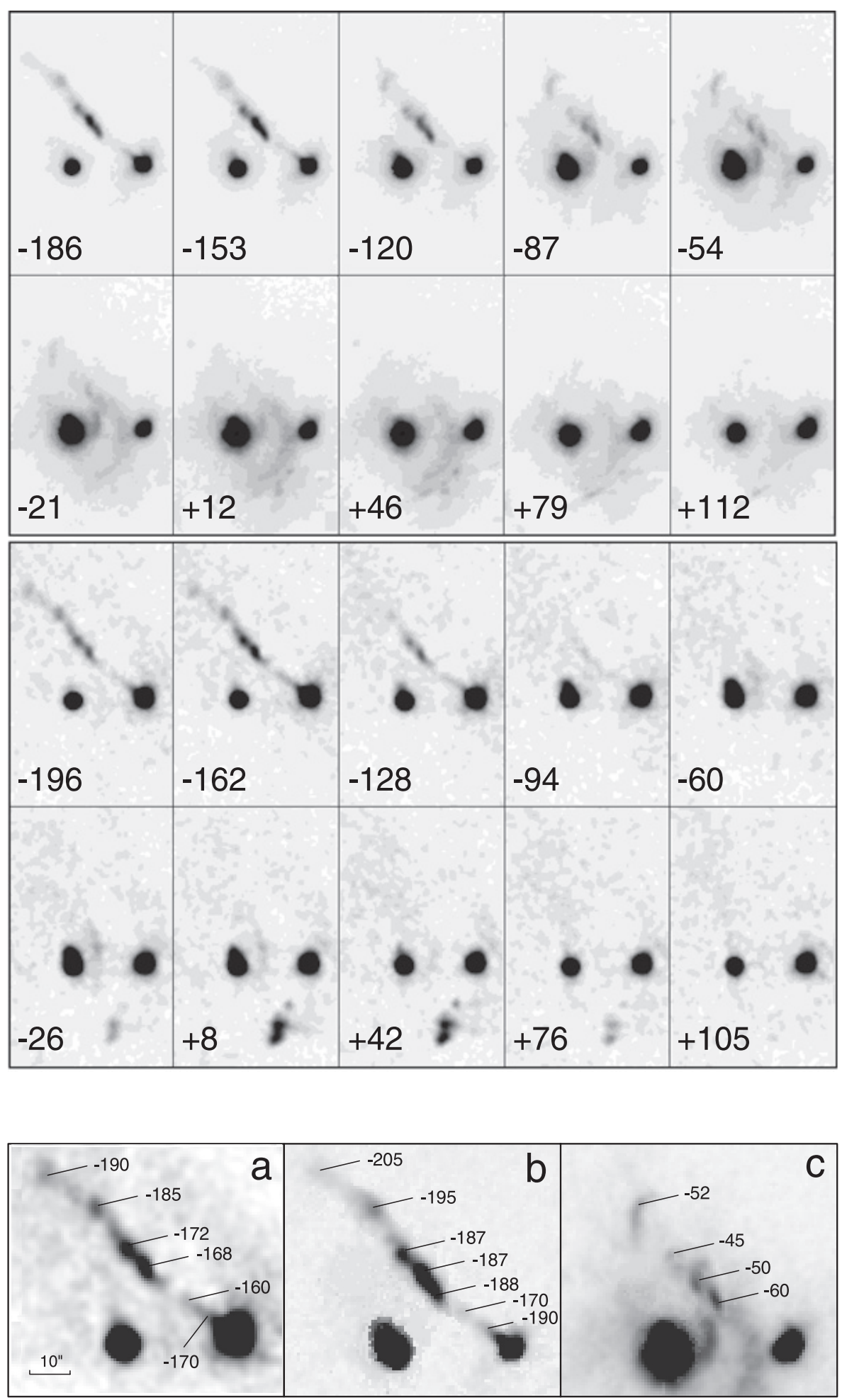

Fig. 2. Channel maps of the HL Tau outflow region covering the $\mathrm{H} \alpha$ (upper panel) and [S II] (lower panel) emission lines. Radial velocities in $\mathrm{km} / \mathrm{s}$ are presented in the lower left corner of each channel. The dimensions of each map are $53^{\prime \prime} \times 84^{\prime \prime}$.

Fig. 3. HL Tau outflow in the high b) and low c) velocity components of $\mathrm{H} \alpha$ emission, as well as in [S II] a). The radial velocities of the knots are shown. They are estimated by Gaussian fitting for each spatial element and averaged for each knot.
The $\mathrm{H} \alpha$ line profiles are split into high- and low-velocity components with mean velocities corresponding to about -150 and $-50 \mathrm{~km} \mathrm{~s}^{-1}$. The velocity of each component undergoes some variation. To investigate these variations in more detail, we separated the two components by Gaussian fitting of each $\mathrm{H} \alpha$ profile from the data cube. After Gaussian fitting (performed by the procedure developed in IDL by A.V.M.), the two separate data cubes were created for the high- and low-velocity components, accordingly. In Fig. 3, the images of the flow in both components, obtained by integrating the intensity in these data cubes, are presented to show the morphology of the two velocity components more clearly.

Using the existing data cubes, it is possible to construct the position-velocity (PV) diagrams with any desired orientation. In Fig. 4 we present such a diagram corresponding to the direction along the HL Tau jet. The positions of the crossing arm structures are marked by arrows. One can see the prominent increase in the velocity dispersion at these places. This diagram agrees with the data obtained by Mundt et al. (1990) (note the ellipsoidal structure of split components) and with the our first 


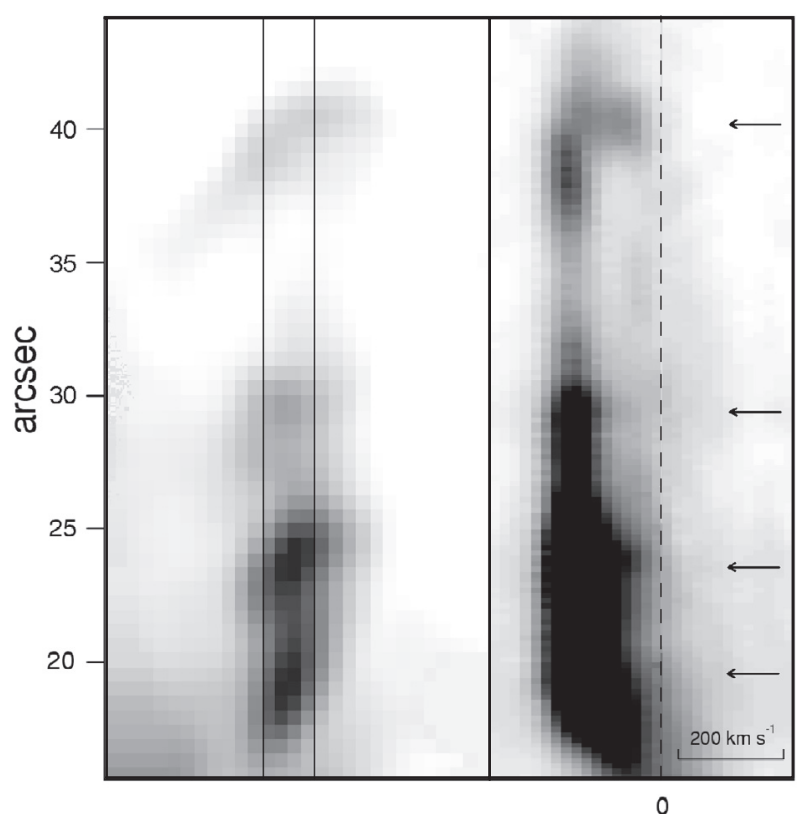

Fig. 4. Position-velocity diagram along the HL Tau jet (right side). The position and dimensions of cut are shown on the left side. The scale to the left shows the distance from the emitting star.

long-slit data obtained with the $6 \mathrm{~m}$ telescope (Magakian et al. 1989), where the split of the $\mathrm{H} \alpha$ profile into the high and low velocity components was detected.

To study the velocity variations perpendicular to the flow, we constructed a PV diagram transverse to the jet in a position of second arcuate structure. The results are shown in Fig. 5 for the observed data cube and for the high- and low-velocity components separately. Also the sum of the two fits is presented to show the quality of the Gaussian fitting. The velocity variation in the high-velocity component (Fig. 5c) perpendicular to the flow is low, with only a small decrease near the edges. In contrast, the velocity gradient in the low-velocity component (Fig. 5d) is about $6 \mathrm{~km} \mathrm{~s}^{-1}$ per arcsec. The velocity measured perpendicular to the axis of the flow (Fig. 6) may indicate either rotation or transverse acceleration.

In the HL Tau star itself the $\mathrm{H} \alpha$ line profile is split into a very broad component with a mean radial velocity of about $+80 \mathrm{~km} \mathrm{~s}^{-1}$ and a narrower and lower-intensity one with a velocity of $-200 \mathrm{~km} \mathrm{~s}^{-1}$. This second component probably traces emission from the jet in the vicinity of the star. This profile agrees with the high-resolution profile presented by Appenzeller et al. (2005).

\subsection{2. [S II] emission}

The velocity channel images of the HL Tau jet in [S II] emission are displayed in the lower part of Fig. 2. Near the base of the jet, they are very similar to those of the $\mathrm{H} \alpha$ line. Several knots belonging to the HL Tau counterjet are also detected in $[\mathrm{S} \mathrm{II}]$ at approximately $+125 \mathrm{~km} \mathrm{~s}^{-1}$. As shown in the work of Mundt et al. (1990) by means of narrow-band imagery, the middle part of the HL Tau jet ("VLA 1 jet") in [S II] emission is narrower than in the $\mathrm{H} \alpha$ line. Our FP observations in [S II] $6716 \AA$ line confirm this result. This line, contrary to the $\mathrm{H} \alpha$ emission, consists of a single-component everywhere in the flow. Moreover, the morphology of the jet in [S II] is very similar to that of the high velocity component of the $\mathrm{H} \alpha$ flow, including the knotty

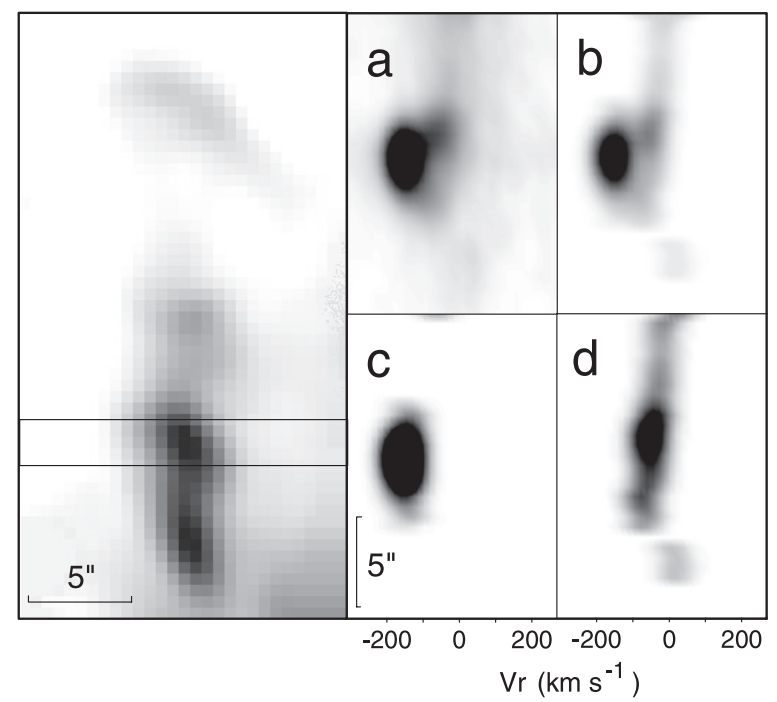

Fig. 5. Position-velocity diagram perpendicular to the HL Tau jet axis (right side). The position and dimensions of cut are shown on the left side. Panel a) is obtained from the observed data cube; panels c) and d) - from the Gaussian fitted high- and low-velocity components; panel $\mathbf{b}$ ) is the sum of $\mathbf{c}$ ) and d) diagrams.

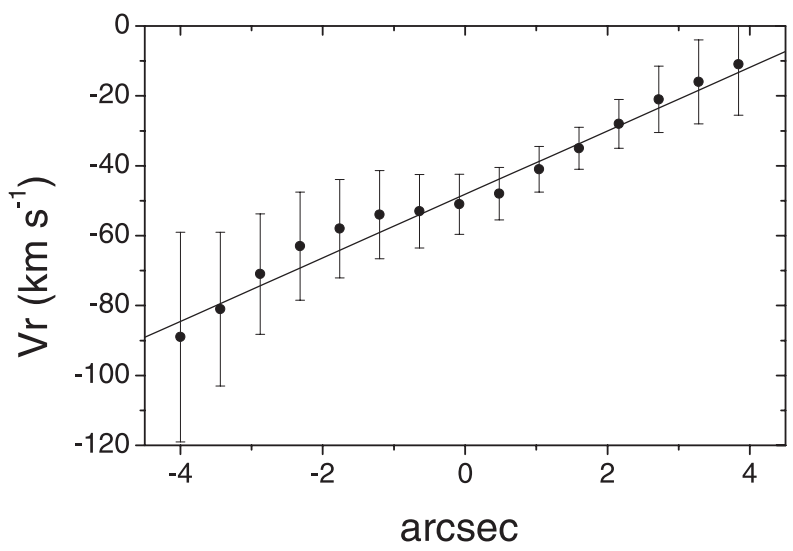

Fig. 6. Radial velocity variation perpendicular to the HL Tau jet axis (from the NW side to SE side of the jet) for the low-velocity component of $\mathrm{H} \alpha$, measured from the PV diagram presented in Fig. 5, d. The zero point of the abscissa corresponds to the jet axis. Note that the intensity peaks in the high- and low-velocity components are slightly shifted, which can be seen from the error bars with highest $\mathrm{S} / \mathrm{N}$.

structures, as shown in Fig. 3. Only the $[\mathrm{S} \mathrm{II}] / \mathrm{H} \alpha$ intensity ratio undergoes some variations.

The most striking feature, however, is the virtually total absence of the arcuate structures in the [S II] line, which are so prominent at low velocities in $\mathrm{H} \alpha$. This is obvious from Figs. 2 and 3.

In the immediate surroundings of HL Tau, the [S II] profiles are split into several components. At the position of the star, the sulphur emission has a double profile with components having radial velocities of about 0 and $-150 \mathrm{~km} \mathrm{~s}^{-1}$, presumably corresponding to emission from the stellar envelope and the base of the jet, respectively. The counter jet emerges at a projected distance of $6^{\prime \prime}$ in the southwest direction from the star with a velocity of about $+140 \mathrm{~km} \mathrm{~s}^{-1}$. Our data are similar to the high-resolution profile of HL Tau [S II] emission shown by Appenzeller et al. (2005). 


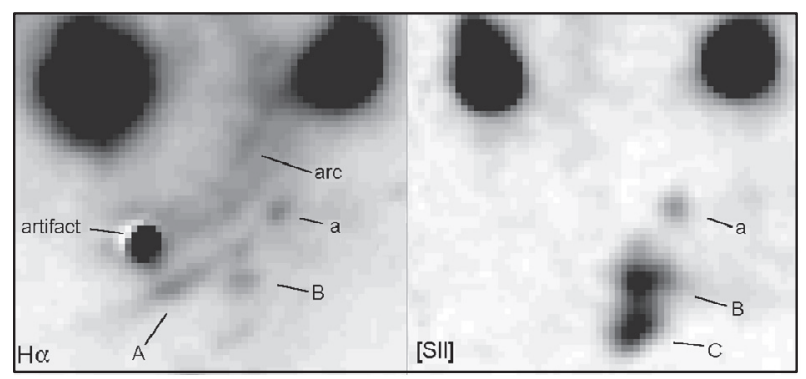

Fig. 7. The southern environment of $\mathrm{HL}$ and XZ Tau in $\mathrm{H} \alpha$ (left panel) and [S II] (right panel) emission, from FP data. Various structures mentioned in the text, including parts of the " $\mathrm{H} \alpha$ jet", are labelled.

\section{2. $X Z$ Tau}

Our data on the bipolar emission nebula associated with XZ Tau are in good agreement with Mundt et al. (1990). A wide opening-angle outflow extends north-northeast with a velocity of $-48 \mathrm{~km} \mathrm{~s}^{-1}$; the [S II] gives nearly the same velocity: about $-40 \mathrm{~km} \mathrm{~s}^{-1}$. A counterflow is seen in [S II], but its faintness makes the line width difficult to estimate. Its radial velocity is about $+125 \mathrm{~km} \mathrm{~s}^{-1}$, which is $50 \mathrm{~km} \mathrm{~s}^{-1}$ higher than in the data of Mundt et al. (1990). The reason for this discrepancy is not clear. No obvious velocity components are seen in either $\mathrm{H} \alpha$ or [S II] profiles of the XZ Tau outflow. However, the line profiles from the star are much wider.

Mundt et al. (1990) noted an unusual structure in both the $\mathrm{H} \alpha$ and [S II] emission NW of XZ Tau. This feature, designated $\mathrm{XZ}-\mathrm{A}$, is fully detached from the star and has the appearance of a curved filament oriented roughly north-south. In [S II], an unresolved knot is detected in the center of this filament and can be clearly seen in the channels corresponding to -26 and $+8 \mathrm{~km} \mathrm{~s}^{-1}$ in Fig. 2. Thus, it appears at near zero velocity and does not display any evidence of bipolarity or other velocity gradients.

Finally, a large $\mathrm{H} \alpha$ arc with a radius of about $20^{\prime \prime}$ is centered on XZ Tau but located to the southwest. This feature touches the HL Tau stellar image and appears to be an $\mathrm{H} \alpha$ reflection nebula (left panel in Fig. 7).

\section{3. "Ho jet"?}

A small group of emission features is located to the southeast of HL Tau and southwest of XZ Tau. These consist of a straight filament of pure $\mathrm{H} \alpha$ emission dubbed the "H $\alpha$ jet" by Mundt et al. (1988) and two knots, marked as "B" and "C" in the same paper, that are visible in both the $\mathrm{H} \alpha$ and [S II] emission lines. These features are shown in Fig. 7.

In addition to having different morphologies and excitation conditions, these features also differ in their radial velocities. Features "B" and " $C$ " and the more compact and fainter knot marked "a" in Fig. 7 have radial velocities in the +21 to $+33 \mathrm{~km} \mathrm{~s}^{-1}$ range in both [S II] and $\mathrm{H} \alpha$ and simple, narrow line profiles. On the other hand, the $\mathrm{H} \alpha$ jet (feature "A") has a wide and asymmetric $\mathrm{H} \alpha$ profile with a radial velocity ranging from +18 to $+46 \mathrm{~km} \mathrm{~s}^{-1}$.

The "A" feature is similar to the arcuate structure southwest of XZ Tau, described at the end of the previous subsection; the two features are nearly parallel and have similar radial velocities. The arcuate structure has a wide $\mathrm{H} \alpha$ profile with a radial velocity of about $+14 \mathrm{~km} \mathrm{~s}^{-1}$. The Mosaic images indicate that this feature marks the border of a zone of higher extinction.

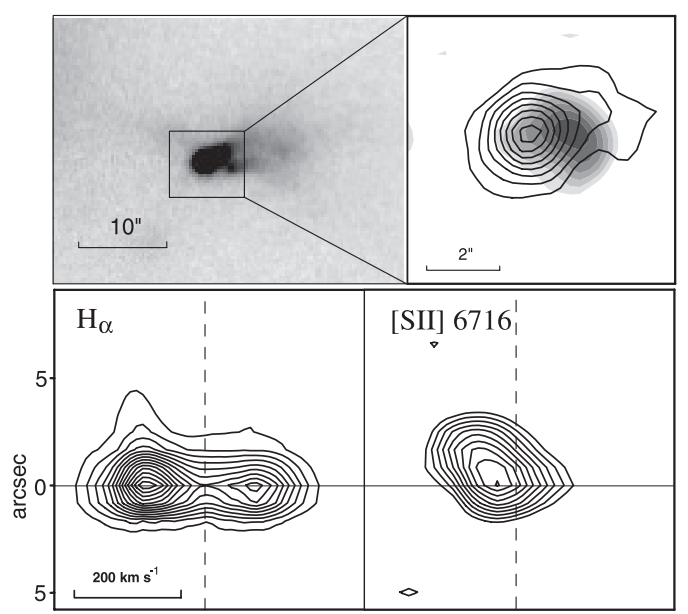

Fig. 8. Upper panel, left - the Mosaic image of $\mathrm{LkH} \alpha 358$ in $\mathrm{H} \alpha$ emission; upper panel, right - restored images of $\mathrm{LkH} \alpha 358$ in [S II] emission (gray scale) and in the corresponding continuum (isolines). Lower panel, left - PV diagram for $\mathrm{H} \alpha$ line in $\mathrm{E}-\mathrm{W}$ direction; right - a similar diagram for the $\lambda 6716[\mathrm{~S} \mathrm{II}]$ emission.

Knots "B" and "C" have a somewhat bow-like appearance and, together with "a", trace another probable outflow. Its source might be one of several very faint stars discovered by Mundt et al. (1988) in deep I images just south of HL Tau. However, it is likely that these stars are unrelated background objects. It is more plausible that these knots trace an outflow emerging from $\mathrm{LhH} \alpha 358$ that is being deflected by the southwestern lobe of the wind emerging from XZ Tau.

\section{4. $L k H \alpha 358$}

This object is also known as CoKu Tau/2 from the list of Cohen \& Kuhi (1979) and represents a low-mass YSO.

The star is associated with a small (about 10") cometary nebula elongated in the eastern direction. The profiles of its $\mathrm{H} \alpha$ emission are split into two prominent components with negative and positive velocities. The negative component is extended in the western direction, which is seen very well in the PV diagram, shown in Fig. 8. The velocities of the components are about $-110 \mathrm{~km} \mathrm{~s}^{-1}$ and $+87 \mathrm{~km} \mathrm{~s}^{-1}$.

Figure 8 also shows restored images of the object in the pure continuum and in [S II] emission obtained after Gaussian fitting and separation of the line and continual emission for each profile. The [S II] images reveal a knot about $1.5-2^{\prime \prime}$ west of the continuum peak representing the star. A PV diagram for [S II] emission is also shown. The line profile is simple, without components, and is blueshifted by $-25 \mathrm{~km} \mathrm{~s}^{-1}$. The extended nature of the [S II] emission is more prominent than in $\mathrm{H} \alpha$. The [S II] radial velocity changes with distance from the source towards the west, reaching up to $-43 \mathrm{~km} \mathrm{~s}^{-1}$. It is interesting that this velocity is lower than that of $\mathrm{H} \alpha$. These features indicate a high-velocity bipolar flow from $\mathrm{LkH} \alpha 358$, perhaps in the form of a micro-jet embedded in the nebula.

\section{5. $\mathrm{HH} 30$}

This object exhibits a long jet and counterjet and is extremely well-studied. Our data confirm the pronounced excitation asymmetry between the jet and counterjet; the jet is bright in [S II] while the counterjet is dominated by $\mathrm{H} \alpha$. The radial velocity data confirm the nearly perfect alignment of the outflow with 


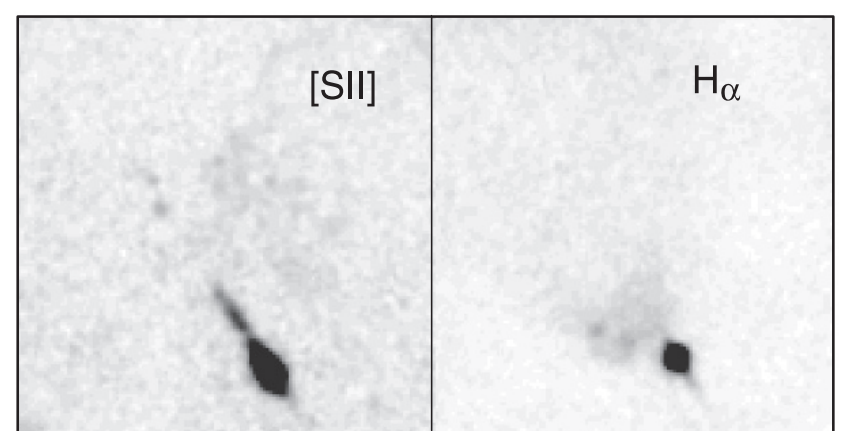

Fig. 9. The $\mathrm{HH} 30$ area in $[\mathrm{S} \mathrm{II}]$ and $\mathrm{H} \alpha$ emissions, images restored from FP data.

the plane of sky. The $\mathrm{H} \alpha$ and [S II] lines have mean radial velocities of $+15 \mathrm{~km} \mathrm{~s}^{-1}$ and $+22 \mathrm{~km} \mathrm{~s}^{-1}$, respectively, close to the $+17 \mathrm{~km} \mathrm{~s}^{-1}$ obtained by Mundt et al. (1990), demonstrating that that our data contain no large systematic errors.

The profile of $\mathrm{H} \alpha$ emission in the direction of the HH 30 star can be divided into two components: a very broad one and the narrow one that corresponds to the jet. We performed Gaussian fitting and found that the broad component has an FWHM of about $230 \mathrm{~km} \mathrm{~s}^{-1}$, while the jet emission has an $F W H M$ of only $70 \mathrm{~km} \mathrm{~s}^{-1}$. The central velocity of the narrow component, of course, is equal to the jet velocity, i.e. about $+15 \mathrm{~km} \mathrm{~s}^{-1}$, while the broad component is probably slightly blueshifted and has nearly zero velocity.

There is a quasi-stellar knot several arcseconds northeast of the HH 30 star (see Fig. 9), described by Mundt et al. (1990), which is visible only in $\mathrm{H} \alpha$. The Mosaic images and FP data show the presence of nebular filaments parallel to the HH 30 outflow. It is possible that this object marks the location of a very faint outflow source (note that in Mundt et al. (1990) it is clearly seen in an I image), a conjecture that requires confirmation.

\section{Results of proper motion measurements}

Figures 10 and 11 show the vectors of proper motion for selected features associated with the HL Tau jet and several emission knots located southeast of HL Tau. Figure 12 shows moving features apparently associated with XZ Tau. Figure 11 also shows a portion of the HH 30 jet that forms a chain of mostly [S II]-dominated knots moving diagonally from the bottom of the image to the upper left. The numerical results of the measurements are given in Table 1.

\subsection{The HL Tau jet}

Figures 10 and 11 and Table 1 confirm that the HL Tau jet is bipolar with proper motions ranging from about 110 to over $200 \mathrm{~km} \mathrm{~s}^{-1}$. (The large motion of box $6,383 \mathrm{~km} \mathrm{~s}^{-1}$, may be spurious due to the low signal-to-noise ratio of this feature.) While the [S II] proper motion vectors point directly away from HL Tau within the measurement uncertainties, the $\mathrm{H} \alpha$ motions along the northeast jet exhibit a systematic deviation from the flow-axis towards the north. In particular, the $\mathrm{H} \alpha$ bow shock located 38" northeast of HL Tau is deflected towards the north by more than $15^{\circ}$ from the average HL Tauri jet direction. This deflection is also apparent in the jet morphology.

The counter jet is very faint, making proper-motion measurements difficult. However, several [S II] knots and one $\mathrm{H} \alpha$ feature exhibit clear motions. The innermost [S II] knot shows an anomalously large motion that may not be real. This feature is very faint and the large apparent motion may be an artifact of brightness variations in the jet and surrounding nebulosity.

\subsection{Southern knots}

The proper-motion data indicate that there are two criss-crossing flows south of HL/XZ Tau. The [S II] knots, representing the part of "H $\alpha$ jet", move towards the southeast. The chain of three knots (the knots "a", "B", and "C", described in Sect. 3.3), has a symmetry axis that passes close to HL Tau, and proper motions indicate general motion away from this star. The proper motions make it clear that the [S II] knots trace parts of an outflow that is distinct from the feature " $\mathrm{A}$ " or the "H $\alpha$ jet". The former knots move towards the southeast away from HL Tau, while the last one moves towards the southwest in a direction away from XZ Tau. This idea is fully supported by the values of the radial velocities of these features.

Inspection of the images and the proper motions of knots "a", "B", and " $C$ " indicate that the velocities decline dramatically towards the southeast. Likewise, the proper motion directions change from fast motion towards the east $\left(\mathrm{PA} \sim 120^{\circ}\right.$ at $V \sim$ $122 \mathrm{~km} \mathrm{~s}^{-1}$ for knot "a") to slow motion towards the south (PA $185^{\circ}$ at $V \sim 12 \mathrm{~km} \mathrm{~s}^{-1}$ for knot "C" - see Table 1). Thus, these three $[\mathrm{S}$ II] knots show pronounced vorticity.

\subsection{The XZTau wind}

The star XZ Tau powers a bubble expanding towards the northeast at PA $\sim 20^{\circ}$ (Krist et al. 1997, 1999). This feature is also apparent in our data. Figure 12 shows the proper motions thought to be associated with outflow from XZ Tau. As mentioned above, our images show that the "H $\alpha$ jet A", located 25" southwest of $\mathrm{XZ}$ Tau is actually a limb-brightened edge expanding away from XZ Tau. Proper motions show that the $\mathrm{H} \alpha$ dominated feature " $\mathrm{A}$ " moves away from XZ Tau and orthogonal to its length. The vector motion in Fig. 12 shows a large westward flow component. However, blinking of the images shows that the motion is orthogonal to the filament. The westward bias may be an artifact of subtle intensity variations in this feature. No well-collimated jet is seen to emerge from the XZ Tau star. The bubble expanding towards the northeast and the $\mathrm{H} \alpha$ feature located southwest indicate the presence of an eruptive, wide-angle wind with an opening angle of about a half radian.

\section{Discussion}

The results presented here confirm that the immediate vicinity of HL and XZ Tau contains at least four outflows: the HL Tau jet, the XZ Tau outflow, the $\mathrm{LkH} \alpha 358$ flow, and also the HH 30 jet. At least one system is a binary (XZ Tau). Other interesting features in this region, such as the presence of the so-called " $\mathrm{H} \alpha$ jet" and the vorticity of the sulphur knots near it, may be explained by the interaction of the XZ Tau counterflow with an outflow from $\mathrm{LkH} \alpha 358$. As discussed above, the new data elucidate some aspects of this interesting system, distinguished by its unusual morphology and kinematics.

\subsection{Interacting outflows and the origin of "Ho jet"}

The morphological and kinematic data provide considerable evidence that we are indeed witnessing the collisions between outflows. This is not surprising given that the outflow from XZ Tau 

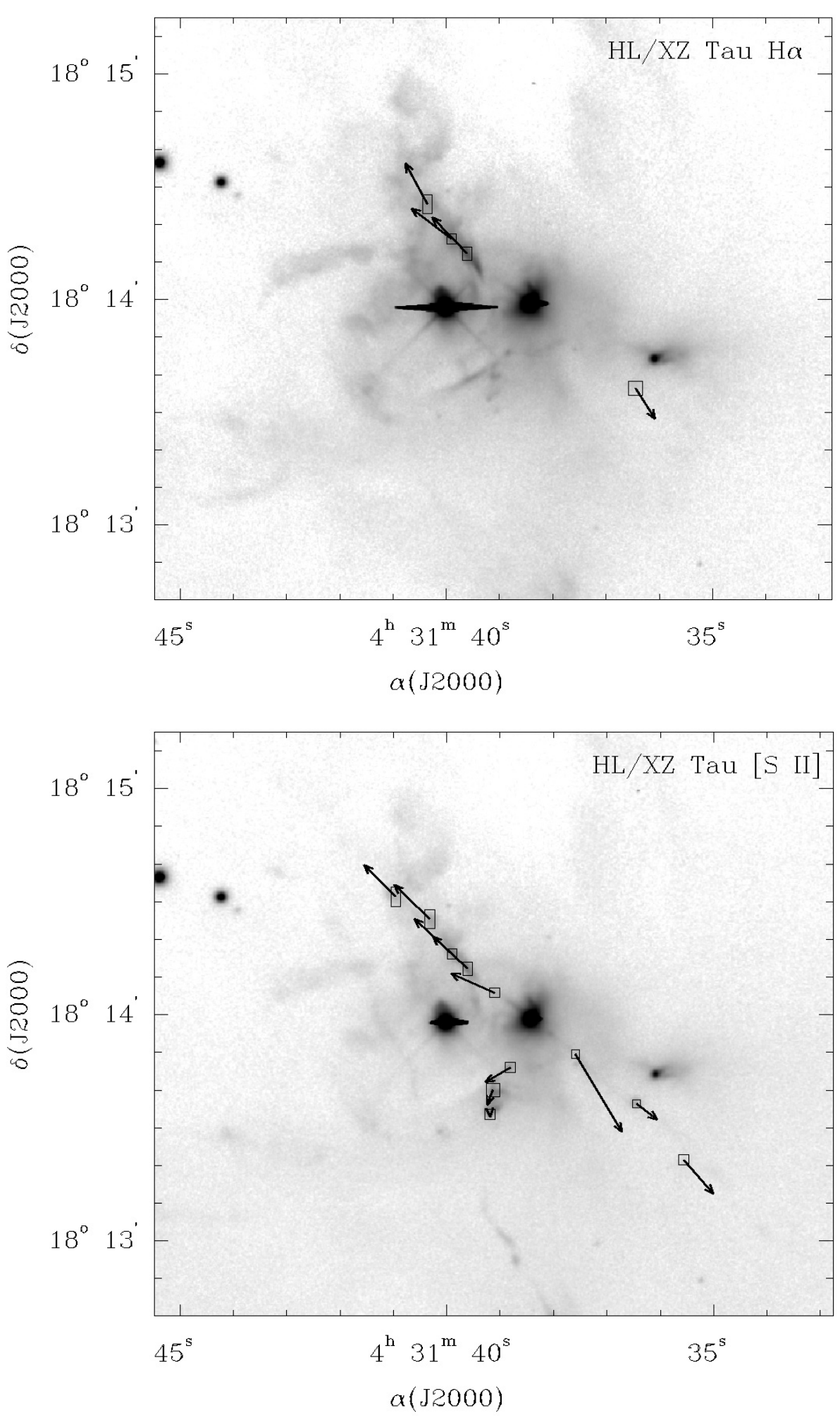

Fig. 10. The proper motions of $\mathrm{H} \alpha$ features near HL Tau, shown in the $\mathrm{H} \alpha$ image, obtained in 2001 with Mosaic.
Fig. 11. The proper motions of [S II] features near HL Tau, shown in the [S II] image, obtained in 2001 with Mosaic. appears to occupy a large surrounding volume making it an obstacle that is hard to avoid.

There are no obvious sources for the [S II] emission knots southeast of HL Tau (B and C in Fig. 7), but there are two possible interpretations for them. First, they could trace a second outflow from a companion to HL Tau that runs almost transverse to the main HL Tau jet. If correct, then either HL Tau must be a close binary system with a companion star lost in the glare of the primary or the companion may be hidden behind the HL Tau disk. The problem with the former picture is that any jet emerging from a close companion to HL Tau must burrow through the thick circumstellar torus known to surround HL Tau without any obvious effects on the structure of this feature, or without producing visible shocks. Another difficulty with such a hypothetical companion star is that there is no evidence of a counterjet.

Perhaps more likely is that the [S II] knots were driven from $\mathrm{LkH} \alpha 358$, located about $35^{\prime \prime}$ west of the knots. As shown in the previous sections, this star drives an outflow oriented roughly in the east-west direction. In this picture, we assume that this 


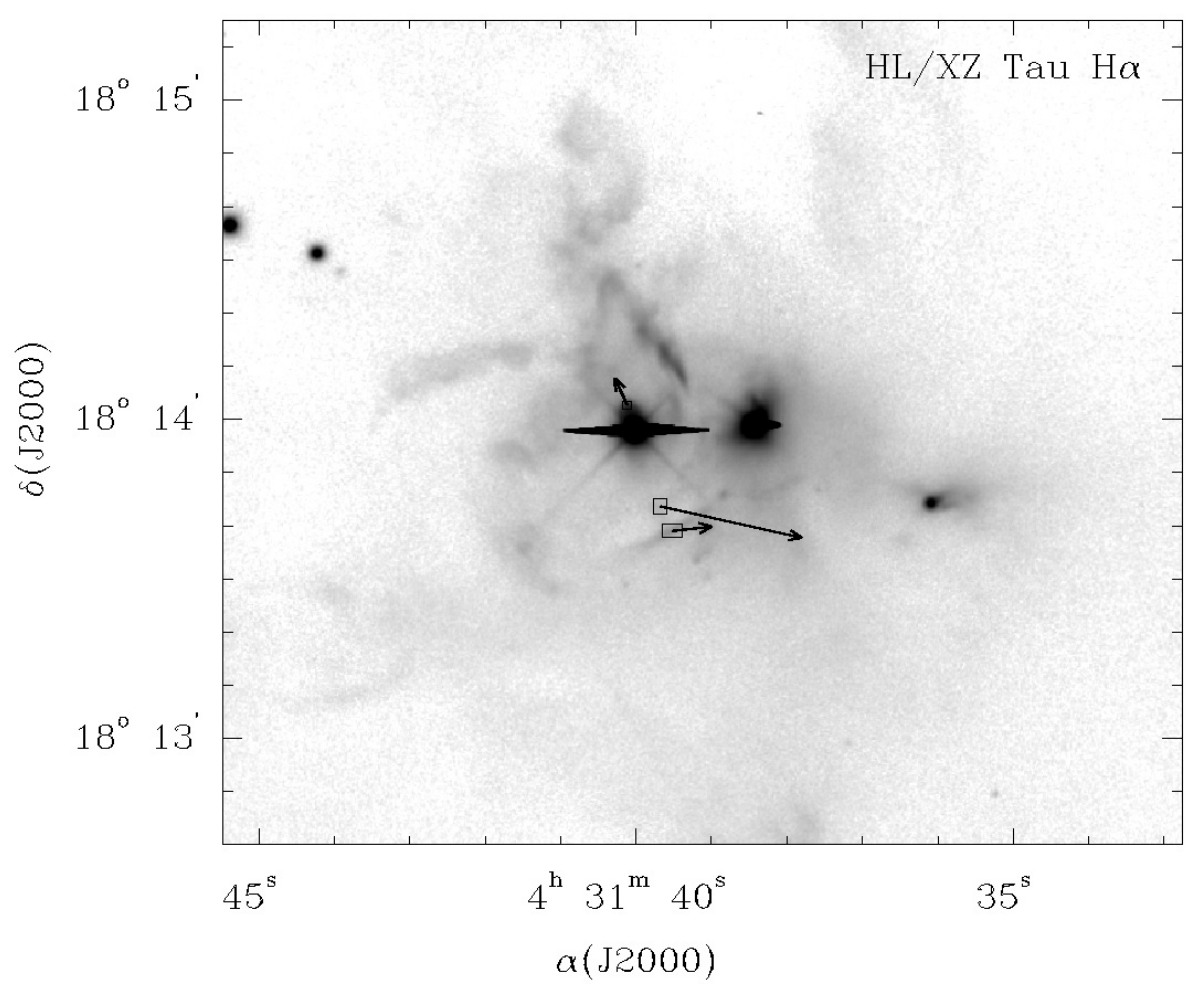

Fig. 12. The proper motions of the features related to XZ Tau. Image is the same as in Fig. 10.

Table 1. Proper motions in the field of HL and XZ Tau.

\begin{tabular}{|c|c|c|c|c|c|c|c|}
\hline Box & $\begin{array}{c}\alpha(\mathrm{J} 2000) \\
\mathrm{h} \mathrm{m} \mathrm{s}\end{array}$ & $\begin{array}{c}\delta(\mathrm{J} 2000) \\
\left.\circ,{ }^{\prime}\right)\end{array}$ & $\begin{array}{l}D \\
\left({ }^{\prime \prime}\right)\end{array}$ & $\begin{array}{c}V \\
\mathrm{~km} \mathrm{~s}^{-1}\end{array}$ & $\begin{array}{c}\text { PA (V) } \\
\left({ }^{\circ}\right)\end{array}$ & $\begin{array}{c}\text { PA (S) } \\
\left(^{\circ}\right)\end{array}$ & $\begin{array}{c}\text { Age } \\
\text { (years) }\end{array}$ \\
\hline & & $\mathrm{H} \alpha$ & & & & & \\
\hline 1 & 43139.65 & 181411.8 & 21.6 & $213.9 \pm 9.8$ & 43.8 & 50.8 & 80 \\
\hline 2 & 43139.94 & 181415.6 & 27.3 & $214.1 \pm 13.5$ & 52.8 & 50.0 & 101 \\
\hline 3 & 43140.39 & 181424.9 & 38.3 & $196.5 \pm 13.4$ & 28.3 & 45.5 & 155 \\
\hline 4 & 43136.49 & $\begin{array}{c}181335.9 \\
{\left[\begin{array}{l}\mathrm{S} \\
\mathrm{II}\end{array}\right]}\end{array}$ & 35.8 & $155.0 \pm 18.2$ & 212.4 & 231.7 & 184 \\
\hline 1 & 43139.14 & 18145.3 & 12.0 & $203.3 \pm 9.8$ & 66.2 & 52.9 & 46 \\
\hline 2 & 43139.65 & 181411.8 & 21.6 & $200.4 \pm 8.1$ & 47.4 & 50.8 & 85 \\
\hline 3 & 43139.94 & 181415.6 & 27.3 & $218.1 \pm 5.4$ & 47.0 & 50.0 & 99 \\
\hline 4 & 43140.35 & 181424.9 & 37.9 & $209.3 \pm 5.9$ & 46.2 & 45.0 & 144 \\
\hline 5 & 43140.99 & 181430.9 & 48.6 & $189.5 \pm 9.5$ & 45.0 & 47.6 & 204 \\
\hline 6 & 43137.64 & 181349.1 & 14.9 & $383.7 \pm 14.3$ & 210.9 & 232.7 & 30 \\
\hline 7 & 43136.49 & 181335.9 & 35.8 & $110.9 \pm 7.2$ & 232.6 & 231.7 & 257 \\
\hline 8 & 43135.61 & 181320.9 & 55.2 & $189.8 \pm 20.6$ & 221.0 & 227.7 & 231 \\
\hline 9 & 43138.85 & 181345.5 & 13.8 & $122.2 \pm 11.6$ & 120.5 & 156.8 & 89 \\
\hline 10 & 43139.18 & 181339.5 & 21.1 & $68.2 \pm 4.1$ & 158.5 & 151.6 & 246 \\
\hline 11 & 43139.23 & 181333.1 & 27.3 & $11.3 \pm 3.3$ & 184.5 & 156.6 & 1913 \\
\hline
\end{tabular}

Column 1: a sequential box number. Unique numbers are assigned if the box centroids or sizes differ significantly for each filter. Columns 2, 3: the J2000 coordinates of each box center. Column 4: the projected distance from the source in arc seconds. Column 5: the proper motion in $\mathrm{km} \mathrm{s}^{-1}$. Column 6: the position angle of the measured velocity vector. Column 7: the position angle of a line drawn from the source to the center of the box. Column 8: the dynamical age of the feature $(\mathrm{D} / \mathrm{V})$ assuming a distance of $140 \mathrm{pc}$.

outflow is sufficiently extended and that its eastern lobe interacts with and is deflected to the south by the southwest-oriented lobe of the bubble created by XZ Tau. If the XZ Tau wind and the eastern lobe of the $\mathrm{LkH} \alpha 358$ outflow lie at the same distance from us, these flows would interact at about the location of the first (northwestern) and fastest proper motion feature located southeast of HL Tau in Fig. 10. Interpenetration of the two flows could explain the rapid decelaration and southward deflection of these knots.

The proper motions and radial velocities provide support for this interpretation. The vectors in Fig. 11 could be interpreted as a vortical motion in a counter clockwise direction and a systematic decrease in velocity from $122 \mathrm{~km} \mathrm{~s}^{-1}$ to under $11 \mathrm{~km} \mathrm{~s}^{-1}$. The radial velocities of the eastern lobe of the $\mathrm{LkH} \alpha 358$ outflow and of the [S II] knots are positive (though they differ by about $50-60 \mathrm{~km} \mathrm{~s}^{-1}$ ), in accordance with the idea that the eastern lobe of this flow is severely decelerated by the southwestern lobe of the wind from XZ Tau.

The western lobe of the $\mathrm{LkH} \alpha 358$ outflow probably propagates towards HH 265, a Herbig-Haro object located about $5^{\prime}$ southwest of $\mathrm{LkH} \alpha 358$ (Devine et al. 1999). There are no other candidate sources to drive this shock, and the orientation of 
its bow is consistent with a flow from the vicinity of $\mathrm{LkH} \alpha 358$. There are two more faint patches visible in $\mathrm{H} \alpha$ on the line connecting $\mathrm{HH} 265$ and $\mathrm{LkH} \alpha 358$.

It is not clear that the deflection of a dense knot or jet is actually being observed. In fact, it would be difficult to deflect a high Mach-number knot through a large angle without the point of deflection being the dominating shock in the region. Instead, the proper motions may belong to the changing points of impact between two outflows, corresponding to the intersection points of two oblique shock waves.

In addition, the interaction may produce the $\mathrm{H} \alpha$ feature known as the $\mathrm{H} \alpha$ jet "A". This arc of emission is elongated orthogonally to the axis connecting it to XZ Tau. It may trace a shock front where the XZ Tau wind either impacts the surrounding medium or where it interacts with the lower-density part of the $\mathrm{LkH} \alpha 358$ outflow that surrounds the [S II] knots. It is difficult to measure the proper motion of this $\mathrm{H} \alpha$ feature. Figure 11 shows our best attempt. Blinking the multi-epoch images shows a clear motion of this feature towards the southwest in the direction of the expected flow from XZ Tau. Our proper motion code, however, has a difficult time constraining the flow vector in the direction along the filament, thus the vectors in Fig. 11 point towards the west. We suspect that this is an artifact of our code and that the motion is actually directly away from XZ Tau.

\subsection{HL Tau jet}

The most striking feature of the HL Tau jet is the unusual dependence of its morphology on radial velocity. At low velocities, the jet exhibits sections of what could be four coils wound on a cone, and the high-velocity narrow channel represents the cone axis. The second important feature is that in [S II] emission the high velocity core is visible only. The understanding of such complex morphology and kinematics is not obvious, but we discuss several explanations below. The rarity of these systems also may be related to the jet-jet interaction.

\subsubsection{Orientation of the jet}

The orientation, $i$, from the line of sight can be derived from the proper motion $U_{\mathrm{pm}}$ and radial velocity $U_{\mathrm{rv}}$ data. Knots of emission moving with speed $v_{\mathrm{k}}=U_{\mathrm{p}} /(\sin i)$ within a jet of speed $v_{\mathrm{j}}=U_{\mathrm{rad}} /(\cos i)$ yields

$\tan i=\frac{v_{\mathrm{j}}}{v_{\mathrm{k}}} \frac{U_{\mathrm{pm}}}{U_{\mathrm{rv}}}$.

The detected axial proper motions of $U_{\mathrm{p}}=210 \pm 10 \mathrm{~km} \mathrm{~s}^{-1}$ (for a distance of $140 \mathrm{pc}$ ) and a radial velocity of $U_{\mathrm{rad}}=180 \mathrm{~km} \mathrm{~s}^{-1}$ yield $i \sim 48-51^{\circ}$ on assuming $v_{\mathrm{k}} / v_{\mathrm{j}}=1$. This implies jet and knot speeds are $\sim 280 \mathrm{~km} \mathrm{~s}^{-1}$.

Radio observations of HL Tau have been interpreted as evidence of a massive dust and gas disk observed at an inclination of about $67^{\circ}$ (Hayashi et al. 1993; Beckwith et al. 1990; Close et al. 1997), surrounded by an extended envelope. Recent modeling of the disc-cavity structure yields a system inclination of $66^{\circ}<i_{\mathrm{d}}<71^{\circ}$, consistent of most, but not all, previous determinations (Lucas et al. 2004). Taking $i \sim 68.5^{\circ}$ as a formal mean value yields $v_{\mathrm{j}} / v_{\mathrm{k}}=2.18$. That is, the knots are moving relatively slowly within a jet of speed $491 \mathrm{~km} \mathrm{~s}^{-1}$. This would imply extremely high-speed shocks, inconsistent with the strong [S II] emission.
An angle close to the plane of the sky would be favored by the almost constant proper motion of the major knots in the jet despite the wiggles of $\sim 5-10^{\circ}$. Then, the high proper motions imply that the jet speed is not constrained to be excessively high and that any change in direction of the knots would not produce large changes in proper motion.

In contrast, the high radial velocities favor motion well out of the sky plane. A plausible model to account for this involves a jet that is deflected. The current jet axis $i \sim 68.5^{\circ}$ that is altered to $i \sim 50^{\circ}$ and diverted by $\sim 15^{\circ}$ towards the north in the sky plane.

\subsubsection{Kinematics and morphology}

We now consider several possible explanations for the HL Tau jet structure. Each accounts for some, but not all, of the characteristics of this jet. First, the sheath morphology can be considered as the main argument supporting the idea of a helical flow around a high-velocity narrow channel. The azimuthal component of this flow would lead to a linear velocity gradient across the jet. A gradient is indeed observed and is almost linear. In addition, to maintain the helical flow, a strong surrounding magnetic field must be invoked, otherwise the jet would expand rapidly and lose all coherent structure (Smith \& Rosen 2007). In fact, the release of a strongly-wound helical field may aid in generating the helical motions. The presence of a non-axial field has been inferred from a polarization analysis (Lucas et al. 2004).

Second, the structure can be interpreted as a series of curved shocks within a sheared jet. A fast jet spine is surrounded by a slower cocoon. The bow shocks are then produced by pulsations originating near the driving star. In this case, we could envision shocks almost co-moving in the jet spine that are, therefore, weak shocks. The flanks of the curved shocks that extend into the slow cocoon would be relatively strong shocks. However, if these bows, driven by ram-pressure, are highly oblique in the cocoon they would not produce excess $\mathrm{H} \alpha$ emission. Furthermore, the proposed jet-cocoon shear entails a low-speed cocoon that would produce a radial velocity gradient rather than a systematic velocity gradient. Nevertheless, the structure of the two leading $\mathrm{H} \alpha$ components shown in Fig. 4 strongly suggests an advancing bow shock driven by the pressure following a Mach disc in the jet.

Many jets have been recorded with multiple bow shocks along them e.g. HH 30 and HH 34. The HL Tau jet is unusual with tilted shocks that become progressively more extended, giving the appearance of a cone. This may be the result of the interaction of the eastern side of the jet with the XZTau bubble, which is the most likely source of the perturbation of the northeast lobe of the HL Tau jet.

HST observations have shown that a bubble of gas is expanding away from XZ Tau towards PA $\sim 20^{\circ}$ (Krist et al. 1999). These features may trace a shock front where a recent increase in the wind speed from XZ Tau is impacting slower moving, older ejecta. The base of the bubble in the HST images appears to subtend a projected opening angle of about a radian as seen from XZ Tau. The western rim of this one radian cone that has an axis at $\mathrm{PA} \sim 20^{\circ}$ intersects the HL Tau jet just at the location of the northward bend where the first of a series of bright knots becomes visible in that flow. We propose that the XZ Tau wind indeed has a projected opening angle of about one radian and that it is responsible for the northward deflection of the HL Tau jet.

Third, a corkscrew structure could be the result of surface wave phenomena, such as fluting modes of the Kelvin-Helmholtz instability (Hardee \& Clarke 1992; Ouyed et al. 2003). In this case, the proper motions may be variable 

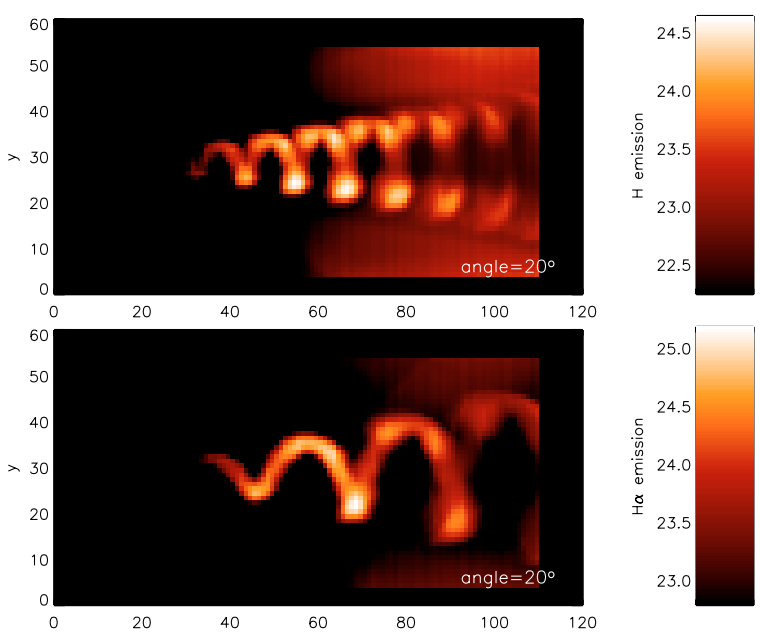

Fig. 13. Hydrodynamic simulations of jets precessing with periods $25 \mathrm{yr}$ (upper panel) and $50 \mathrm{yr}$ (lower panel) and $10^{\circ}$ opening half-angle. The images display the predicted $\mathrm{H} \alpha$ emission at the indicated angle relative to the plane of the sky at a time of 1250 years after the jet was injected (from a star located at $x=25, y=30$ ). Parameters are: grid size: $50 \times 50 \times 100$, zone size: $2 \times 10^{15} \mathrm{~cm}$, jet radius $6 \times 10^{15} \mathrm{~cm}$, ambient and jet densities of 10 and $300 \mathrm{~cm}^{-3}$, jet speed of $300 \mathrm{~km} \mathrm{~s}^{-1}$, initial jet temperature $6000 \mathrm{~K}$. A screen with optical depth of $10 \exp (-x / 2 \times$ $10^{16} \mathrm{~cm}$ ) was applied to simulate the inner obscuration.

and considerably less than fluid speeds, depending on the relative density across the surface. The jet gas could be deflected or twisted to produce azimuthal motions. This again requires a twocomponent jet, with an outer slower sheath interacting with a fast spinal jet.

Fourth, a fast precessing jet could drive a helical flow that maintains its basic flow structure when ballistic. The jet helix drives a fast helical shock into ambient gas and is itself radiative due to weak internal shocks. In this model, the gradient in radial velocity can be the consequence of a gradual change in jet orientation and direction in the plane of the sky. If the angle $i$ changes from $60^{\circ}$ to $75^{\circ}$, then a sheath of speed $\sim 140 \mathrm{~km} \mathrm{~s}^{-1}$ could exhibit the observed velocity gradient. To gain insight, we performed a sequence of simulations of fast-precessing hydrodynamical jets, following on the work of Raga et al. (1993) and Rosen \& Smith (2004). We find that a wide range in precession and pulsation rates produces chains of arcs and knots, although no convincing velocity structure was uncovered. The best results were obtained for a simple precessing jet and, as displayed in Fig. 13, the arcs observed in $\mathrm{H} \alpha$ are roughly reproducible for an orientation of $20^{\circ}$ out of the sky plane, consistent with the disk axis orientation but not the high radial velocity. Moreover, a rapid precession period is necessary to produce a tightly wound coil.

\section{Conclusion}

We summarize our findings as follows.

HL Tau ejects a bipolar jet with unusual bends and a twocomponent structure in the northern jet. Its morphology and kinematics strikingly differ in $\mathrm{H} \alpha$ and [S II] emission. Likewise, the $\mathrm{LkH} \alpha 358$ star also drives a bipolar jet that is detected in the immediate vicinity of the star and also appears to produce an extended outflow.

A number of reflection filaments and emission knots in the environments of the HL/XZ Tau pair were studied. Their origin is probably connected with a wide-angle wind emerging from XZ Tau. Its southwestern lobe creates a shock that appears to be responsible for the "H $\alpha$ jet", a reflection-emission ridge elongated orthogonally to the flow direction from this star. This structure exhibits motion away from XZ Tau. Meanwhile, the highly collimated northeastern lobe of the jet from HL Tau is deflected towards the north about $20^{\prime \prime}$ northeast of the source star, probably by the northeastern lobe of the XZ Tau wide-angle wind.

The [S II] knots located several arcseconds southwest of the "H $\alpha$ jet" filament move toward the east and southeast and may trace the location where the southwestern lobe of the XZ Tau wind deflects a collimated flow from $\mathrm{LkH} \alpha 358$. Thus, these knots and the "H $\alpha$ jet" may trace yet another collision between a collimated outflow and a wide-angle wind. The rarity of jets with such complex velocity structure is thus due to the particular conditions: a tight nest of jets and winds.

The high and uniform proper motions in the HL Tau jet suggest that the weak shocks within it are almost co-moving. The shocks are likely to be maintained by the ram pressure of a lowdensity crosswind from XZ Tau. This results in a sheath containing fast-moving oblique shocks of intermediate strength.

The remarkable jet-wind collisions in the HL/XZ Tau region provide evidence that some young stars produce wide-angle winds. XZ Tau is the least-obscured YSO that is associated with an outflow in this region. It is possible that the collimation of an outflow depends on the evolutionary state of the source YSO. As the disk and other circumstellar matter are dispersed, collimation of an outflow declines.

Acknowledgements. This project was supported by INTAS grant 03-51-4838 and by the grant CRDF/NFSAT AS062-02/CRDF 12009. It was also partially supported by ANSEF grant 05-PS-astroex-0719-132.

\section{References}

Afanasiev, V. L., \& Moiseev, A. V. 2005, Astron. Lett., 31, 193 Appenzeller, I., Bertout, C., \& Stahl, O. 2005, A\&A, 434, 1005 Beckwith, S. V. W., Sargent, A. I., Chini, R. S., \& Guesten, R. 1990, AJ, 99, 924 Brown, A., Drake, S. A., \& Mundt, R. 1985, in Radio Stars, ed. R. M. Hjellming, \& D. M. Gibson, Reidel, Dordrecht, 105

Close, L. M., Roddier, F., Northcott, M. J., et al. 1997, ApJ, 478, 766

Cohen, M., \& Kuhi, L. V. 1979, ApJS, 41, 743

Devine, D., Reipurth, B., \& Bally, J. 1999, AJ, 118, 972

Hardee, P. E., \& Clarke, D. A. 1992, ApJ, 400, L9

Hartigan, P., Morse, J. A., Reipurth, B., Heathcote, S., \& Bally, J. 2001, ApJ, 559, L157

Hayashi, M., Ohashi, N., \& Miyama, S. M. 1993, ApJ, 418, L71

Krist, J. E., Burrows, C. J., Stapelfeldt, K. R., et al. 1997, ApJ, 481, 447

Krist, J. E., Stapelfeldt, K. R., Burrows, C. J., et al. 1999, ApJ, 515, L35

Lopez, R., Raga, A. C., Riera, A., et al. 1995, MNRAS, 274, L19

Lopez, R., Riera, A., Raga, A. C., et al. 1996, MNRAS, 282, 470

Lucas, P. W., Fukagawa, M., Tamura, M., et al. 2004, MNRAS, 352, 1347

Magakian, T. Yu., Movsessian, T. A., Afanasiev, V. L., \& Burenkov, A. N. 1989, Sov.Astr.Lett., 15, 53

Moiseev, A. V. 2002, Bull. SAO, 54 [arXiv: astro-ph/0211104]

Mundt, R., \& Fried, J. W. 1983, ApJ, 274, L83

Mundt, R., Brugel, E. W., \& Bührke, T. 1987, ApJ, 319, 275

Mundt, R., Ray, T., \& Bührke, T. 1988, ApJ, 333, L69

Mundt, R., Ray, T. P., Bührke, T., Raga, A. C., \& Solf, J. 1990, A\&A, 232, 37

Ouyed, R., Clarke, D. A., \& Pudritz, R. E. 2003, ApJ, 582, 29

Raga, A. C., Canto, J., \& Biro, S. 1993, MNRAS, 260, 1632

Reipurth, B., Heathcote, S., Morse, J., Hartigan, P., \& Bally, J. 2002, AJ, 123, 362

Rodríguez, L. F., Cantó, J., Torrelles, J. M., Gómez, J. F., \& Ho, P. T. P. 1992, ApJ, 393, L29

Rodríguez, L. F., Cantó, J., Torrelles, J. M., et al. 1994, ApJ, 427, L103

Rosen, A., \& Smith, M. D. 2004, MNRAS, 347, 1097

Smith, M. D., \& Rosen, A. 2007, MNRAS, submitted

Wilner, D. J., Ho, P. T. P., \& Rodríguez, L. F. 1996, ApJ, 470, L117 